\title{
Hysteresis Nutation Damper for Spin Satellite
}

\author{
Hamed Shahmohamadi Ousaloo*
}

Space Science Research Institute (SSRI), Tehran, Iran

\begin{abstract}
Hysteresis dampers are commonly used in Passive magnetic Attitude Control System (PACS). In PACS these rods produce a damping torque and reduce the satellite angular momentum and angular velocity. In this paper, a spin satellite was investigated which utilizes a passive magnetic damper consisting of magnetic hysteresis rods aligned with principal axis or spin axis of satellite and de-tumbling of the satellite, and the pure spin was achieved.

An analytical model was presented to analyze hysteresis damper and a numerical simulation was performed to obtain dynamic properties of the spin attitude. In addition, assuming a dynamic imbalance, attitude behavior and damper effect on the spin rate of satellite were analyzed. The behavior of this passive magnetic stabilized satellite was simulated from the initial post separation phase.
\end{abstract}

Keywords: Hysteresis dampers, nutation damper, spin stabilization, dynamic imbalance, passsive magnetic attitude control system.

\section{INTRODUCTION}

Spin stabilization is a current method to stabilize the attitude of a satellite in space. With spin stabilization, the satellite gains a gyroscopic stiffness which makes its accurate control possible. Unwanted oscillations of the satellite must be damped out. Therefore, satellites are usually equipped with one or more nutation damping equipments. Nutation or damping unwanted angular rate can be achieved by active or passive attitude control. The active way is performed by countering the attitude determination using the respective sensors. This feedback system increases the power consumption, complexity, and risk of an active attitude determination and control system. But in the passive way, no external energy and additional sensors and actuators are required. However, a dynamic analysis of the satellite attitude with damper is required to obtain mass properties and arrangement of the damper inside the satellite without any damage.

Passive angular rate damping of the satellite using dampers was first performed in 1963 by Miles [1]. Many types of nutation dampers have been designed for spinstabilized spacecrafts ranging in size from small to large satellites. Nutation dampers dissipate the kinetic energy of periodic rotations of a satellite in a specific direction. When energy is dissipated, the principal axis of the angular momentum vector becomes aligned with the largest moment of inertia. There are a variety of these dampers including viscous ring dampers [2], ball in tube dampers [3], pendulum dampers [4], wheel dampers [5] and spring-mass-dampers [6].

*Address correspondence to this author at the Space Science Research Institute (SSRI), Tehran, Iran; Tel:+982177919668; Fax;+982177919668; Email: hamed.oosaloo@gmail.com
The purpose of the present paper is to investigate the results of using hysteresis dampers as nutation dampers in spin satellite to dissipate kinetic energy. One way to acquire passive angular rate damping is simply adding magnetic hysteresis material. Passive magnetic stabilization is very attractive. It's often used in small and light satellites to gain basic pointing or merely to avoid random and unpredictable tumble. Magnetic hysteresis rods are used to create passive de-tumble torques on the satellite. With this system, the rotation is damped about two un-spin axes. The main advantages of these dampers are their cost and reliability. But they are not programmable and their capability for attitude stabilization is limited.

\section{DESIGN OF HYSTERESIS DAMPER FOR SPIN SATELLITE}

To investigate hysteresis damper, a mathematical simulation of the hysteresis phenomenon and the satellite spin is required. Hysteresis dampers are currently used in passive magnetic attitude control system (PACS) [7-9]. PACS has two main components. One plays as permanent magnets which aligns the satellite with the earth magnetic field when it moves on its orbit. These permanent magnets are made of hard ferromagnetic materials and exchange the angular momentum of the satellite. The other component of PACS is a set of hysteresis rods. These rods produce a damping torque to enhance the energy dissipation property of soft ferromagnetic materials and reduce the satellite angular momentum by converting a part of the angular motion kinetic energy to heat; consequently, the angular velocity decreases. Hysteresis magnetic materials are much like permanent magnets in their function, except that their permeability is significantly higher. The most popular and current hysteresis magnetic material is hysteresis damper represented by an elongated rod made of soft-magnetic materials with heat treatment. Hysteresis materials have 
magnetic domains with random distribution resulting in a zero magnetic dipole. When subjected to an external field, the domains orient themselves. After removing the external field, the residual magnetization remains. In fact, depending on the material magnetic properties, it retains a magnetic dipole of some strength when the external magnetic field is removed.

The damping torque provided by the hysteresis rods in a magnetic field is obtained from:

$T=m \times B^{b}$

where $\mathbf{B}^{\mathbf{b}}=\left[\mathbf{B}_{\mathbf{x}}{ }^{\mathbf{b}} \mathbf{B}_{\mathbf{y}}{ }^{\mathbf{b}} \mathbf{B}_{\mathbf{z}}{ }^{\mathbf{b}}\right]$ is the earth magnetic flux expressed in body-fixed frame relative to inertial frame, $\mathbf{m}$ is the magnetic moment of the hysteresis rod given by:

$m=\left[\begin{array}{lll}m_{h} & 0 & 0\end{array}\right]$

$m_{h}=\frac{B_{h} V_{h}}{\mu_{0}}$

where $\mathbf{m}_{\mathbf{h}}$ is the magnetic moment of hysteresis rod aligned with spin axis or $X$ axis (Fig. 1), $\mathbf{B}_{\mathbf{h}}$ is the magnetic flux induced in the rod, $\mathbf{V}_{\mathbf{h}}$ is the volume of the rod, and $\mu_{0}$ is the permeability of free space.

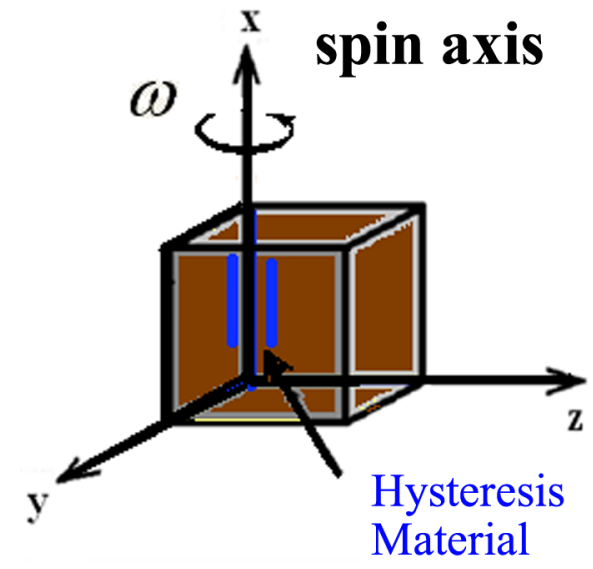

Fig. (1). Hysteresis materials in spin satellite aligned with spin axis.

In this paper, hysteresis damper was investigated with two hysteresis rods aligned with the satellite spin axis (Fig. 1). In this configuration, the un-spin angular rate is damped passively and the nutation angle is dissipated.

The hysteresis rods produce variable magnetic dipoles $\left(\mathbf{m}_{\mathbf{h}}\right)$ proportional to the earth magnetic field component along with the satellite spin axis. Hysteresis magnetic materials have significantly higher permeability than permanent magnets. Hence, affecting by a variable magnetic field, the hysteresis materials tend to show a dynamic realignment of micro-magnetic dipoles and a variation in magnetic domain boundaries. These changes cause frictional dissipation of energy at the molecular level. This phenomenon is known as hysteresis dissipation [13].

\section{MODEL OF MAGNETIC HYSTERESIS DAMPING}

The B-H curve of Fig. (2) represents a hysteresis loop at saturation state. This loop is generally defined by three magnetic hysteresis parameters. The material can be characterized by the maximum magnetization (saturation induction (Bs)), the remaining magnetization after removal of the external field (remanence $(\mathbf{B r})$ ), and the magnetic field required to nullify the magnetization (coercive force $(\mathbf{H c})$ ). The way by which soft magnetic materials are magnetized depending on the external field can be displayed in a B-H curve.

This curve was shown in Fig. (2).

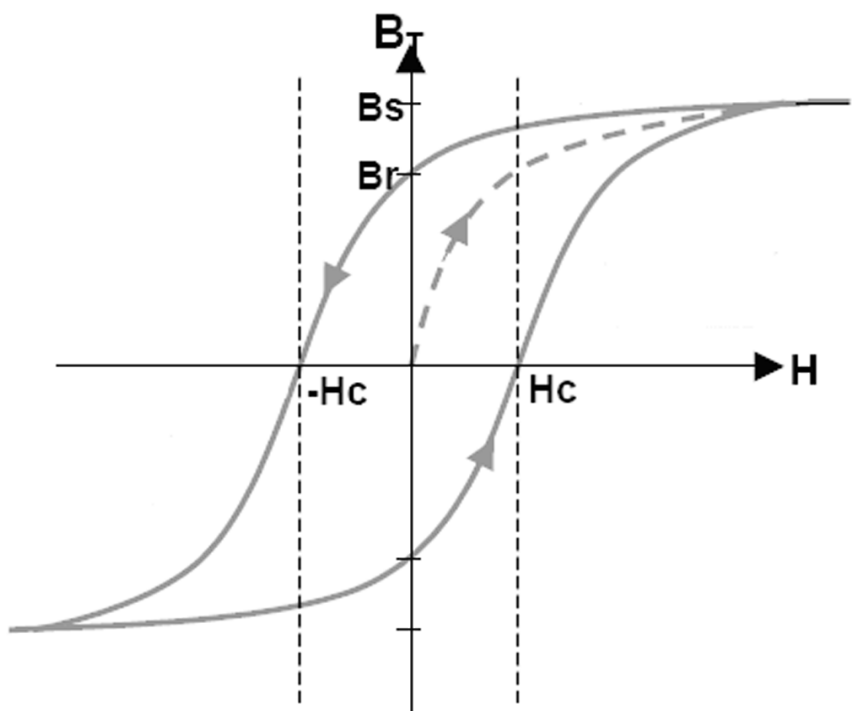

Fig. (2). Hysteresis loop diagram.

Various mathematical models were presented for hysteresis rods in the literature $[10,11]$. One of them was proposed by Kumar [11] based on an induced flux density developed by Flately [10] as:

$$
\begin{aligned}
& \gamma=\frac{1}{H_{C}} \tan \left(\frac{\pi B_{r}}{2 B_{s}}\right) \\
& B_{h}=\frac{2}{\pi} B_{s} \tan ^{-1}\left[\gamma\left(H \pm H_{c}\right)\right]
\end{aligned}
$$

where $\gamma$ is a constant value and $\mathbf{H}$ is the component of magnetic field strength aligned with the hysteresis rod. A positive value for Hc is used if $\mathbf{d H} / \mathbf{d t}<\mathbf{0}$ and a negative value of $\mathbf{- H c}$ is used if $\mathbf{d H / d t}>\mathbf{0}$.

The magnetic material PERMENORM $5000 \mathrm{H} 2$ is used in all hysteresis rod dampers because it produces high hysteresis losses and is unaffected by pace environment over long periods of time. Some properties of this material are:

\section{$H_{c}[A / m]=5, B_{s}[T]=1.55, B_{r}[T]=0.755, \rho\left[g / \mathrm{cm}^{3}\right]=8.25$ \\ HYSTERESIS DAMPER AND SPIN DYNAMIC CONDITIONS}

Consider the rotational properties of a rigid satellite equipped with hysteresis dampers. First, we assume that the satellite is dynamically balanced and the body-fixed coordinates are selected to be coincident with the spacecraft principal axes. The dynamic properties of spacecraft are given by:

$d L_{B} / d t=N_{B}-\omega_{B I} \times L_{B}$ 
$d L_{I} / d t=N_{I}=A_{B I}^{T} N_{B}$

where $\mathbf{L}_{B}$ is the spacecraft angular momentum about center of mass expressed in the spacecraft body frame or in inertial reference frame, $\mathbf{L}_{\mathrm{I}}$. $\mathbf{N}$ is the external torque including the disturbance torques and the hysteresis torques. $\mathbf{A}_{\mathbf{B I}}$ is the inertial-to-body attitude matrix. The angular velocity $\boldsymbol{\omega}_{\mathbf{B I}}$ is given by:

$\omega_{B I}=J^{-1} L_{B}$

where $\mathbf{J}$ is the spacecraft moment-of-inertia tensor $\left(\mathrm{kgm}^{2}\right)$.

Spacecraft dynamic condition is usually modeled by Eq. (4a) and kinematic condition is modeled by:

$d A_{B I} / d t=-\left[\omega_{B I} \times\right] A_{B I}$

where

$$
[\gamma \times]=\left[\begin{array}{ccc}
0 & -\gamma_{3} & \gamma_{2} \\
\gamma_{3} & 0 & -\gamma_{1} \\
-\gamma_{2} & \gamma_{1} & 0
\end{array}\right]
$$

A quaternion or some other lower-dimensional representation of $\mathbf{A}_{\mathbf{B I}}$ is often integrated rather than Eq. (6). In this paper, the kinematic equations were expressed by separate integrations of the vector and the scalar part of the attitude quaternion.

$$
\left[\begin{array}{c}
\dot{\varepsilon}_{1} \\
\dot{\varepsilon}_{2} \\
\dot{\varepsilon}_{3} \\
\dot{\eta}_{4}
\end{array}\right]=1 / 2\left[\begin{array}{cccc}
0 & \omega_{z} & -\omega_{y} & \omega_{x} \\
-\omega_{z} & 0 & \omega_{x} & \omega_{y} \\
\omega_{z} & -\omega_{x} & 0 & \omega_{z} \\
-\omega_{x} & -\omega_{y} & -\omega_{z} & 0
\end{array}\right]\left[\begin{array}{c}
\varepsilon_{1} \\
\varepsilon_{2} \\
\varepsilon_{3} \\
\eta_{4}
\end{array}\right]
$$

where the quaternion $q=\left(\varepsilon_{1}, \varepsilon_{2}, \varepsilon_{3}, \eta_{4}\right)$ for attitude representation can be derived from the Euler axis, e, and principal rotation angle, $\boldsymbol{\theta}$, as follows:

$\varepsilon_{1}=e_{1} \sin (\theta / 2)$

$\varepsilon_{2}=e_{2} \sin (\theta / 2)$

$\varepsilon_{3}=e_{3} \sin (\theta / 2)$

$\eta_{4}=\cos (\theta / 2)$

A quaternion satisfied the constraint $q^{T} q=1$ :

$\eta^{2}+\varepsilon_{1}^{2}+\varepsilon_{2}^{2}+\varepsilon_{3}^{2}=1$

Hysteresis dampers utilize the magnetic rate damping to establish a desired spin rate about the spin axis or $X$ axis and remove transverse rates about the $\mathrm{Y}$ and $\mathrm{Z}$ axes. A Lyapunov function can be used in the form:

$V=1 / 2\left(\omega-\omega_{s}\right)^{T} I\left(\omega-\omega_{s}\right)$

where

$$
\omega_{s}=\left[\begin{array}{lll}
\Omega & 0 & 0
\end{array}\right]
$$

where $\boldsymbol{\omega}$ is the rate vector, $\boldsymbol{\omega}_{\mathrm{s}}$ is the desired rate vector about $\mathrm{X}$ axis, and $\boldsymbol{\Omega}$ is the acquisition spin rate. It can be shown that if the magnetic hysteresis is selected based on Eq. (2), the time variation rate of $\mathbf{V}$ is negative for an axisymmetric inertia matrix of the form $\mathbf{I}=\operatorname{diag}\left(\mathbf{I}_{\mathrm{s}}, \mathbf{I}_{\mathbf{t}}, \mathbf{I}_{\mathbf{t}}\right)$. It was observed in Fig. (3) that the time rating of Lyapunov function is negative throughout nutation damping via hysteresis rods.

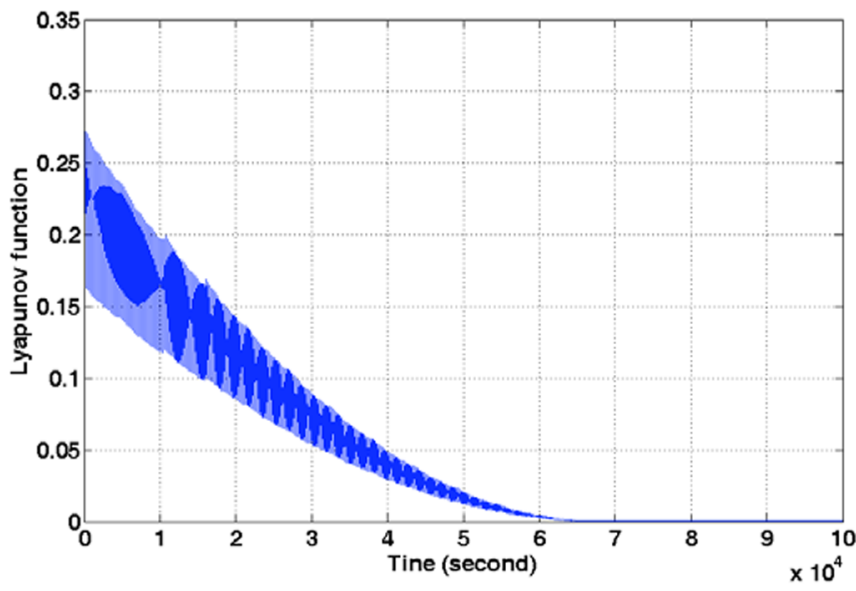

Fig. (3). Lyapunov function.

The imbalance existing in a spinning body can be in the form of static or dynamic imbalance. A static imbalance is generally an imbalance in a radial direction to the axis of rotation and is produced by a force that remains constant in the orientation relative to the spinning body. On the other hand, a dynamic imbalance is generally produced by a moment created when assembly spins rotate about an axis other than the principle axis. When an asymmetric body rotates about the principle axis, or a symmetric body rotates about an axis other than the principle axis, the outcome is dynamic imbalance. Due to the dynamic imbalance, the spinning body develops an angle of wobble at which the angular momentum vector aligns with the axis with the maximum moment of inertia; although this vector doesn't coincide with the spin axis. In fact, in this situation, the rotating object possesses product of inertia in the body frame system. Since the nutation angle is the angle between the angular momentum vector and the spin axis of the spacecraft, in the balanced case, the spin axis and the principal axis coincide, and the nutation is formed because of disturbance and the initial angular velocity in post separation phase. However, under imbalanced condition, since the angular momentum axis and the spin axis do not align, nutation (which is known as wobble angle) always exists [12]. Considering that the spacecraft angular momentum is co-aligned with the satellite principal axis, the direction of the spacecraft major axis is different from the direction of spin axis or hysteresis rods due to the nature of imbalance. Also, for an unbalanced spacecraft, the angular momentum is expressed as:

$\left[\begin{array}{c}\chi_{x} \\ \chi_{y} \\ \chi_{z}\end{array}\right]=\left[\begin{array}{l}I_{x x} \omega_{x}-I_{x y} \omega_{y}-I_{x z} \omega_{z} \\ I_{y y} \omega_{y}-I_{x y} \omega_{x}-I_{y z} \omega_{z} \\ I_{z z} \omega_{z}-I_{y z} \omega_{y}-I_{x z} \omega_{z}\end{array}\right]$ 
Consequently, for the nutation angle, we have:

$$
\begin{aligned}
& \theta=\tan ^{-1}\left(\frac{\sqrt{\chi_{y}^{2}+\chi_{z}^{2}}}{\chi_{x}}\right)= \\
& \tan ^{-1}\left(\frac{\sqrt{\left(I_{y y} \omega_{y}-I_{x y} \omega_{x}-I_{y z} \omega_{z}\right)^{2}+\left(I_{z z} \omega_{z}-I_{y z} \omega_{y}-I_{x z} \omega_{z}\right)^{2}}}{I_{x x} \omega_{x}-I_{x y} \omega_{y}-I_{x z} \omega_{z}}\right.
\end{aligned}
$$

where $\chi=\left[\begin{array}{lll}\chi_{x} & \chi_{y} & \chi_{z}\end{array}\right]$ is the angular momentum expressed in body-fixed frame relative to inertial frame.

\section{SIMULATION RESULTS}

An effective attitude dynamic design begins with an analysis of external torques by the satellite. The satellite experiences these torques at the height of 500 kilometers. The simulation was for an oblate spin satellite with the following mass properties: bus mass $\mathbf{m}_{\mathbf{b}}=40 \mathrm{~kg}$, with principal moments of inertia $\left(\mathrm{kg} \cdot \mathrm{m}^{2}\right)$ of $\mathbf{I x x}=0.750$, $\mathbf{I y y}=0.735$, $\mathbf{I z z}=0.725$. Under dynamically unbalanced situation, the product of inertia is $\mathbf{I x y}=\mathbf{I} \mathbf{x z}=\mathbf{I y z}=0.01$; these amounts are zero for balanced satellite. The IGRF model for the earth magnetic field is incorporated into the computation of the magnetic torques due to the hysteresis rods. Two hysteresis rods were assumed to be identical, with volume $\mathbf{V}_{\mathbf{h}}=8.75 \times 10^{-7} \mathrm{~m}^{3}$ and hysteresis constants as stated earlier. The initial angular velocity at post separation phase was $\omega(0)=\left[\begin{array}{llll}30 & 30 & 30\end{array}\right] \mathrm{deg} / \mathrm{sec}$.

A complete simulation of the satellite during the first 16 orbits after separation was illustrated in Figs. (4-8), including plots of the body angular rates $\left(\omega_{\mathrm{x}}, \omega_{\mathrm{y}}, \omega_{\mathrm{z}}\right)$ for dynamically balanced satellite (Figs. 4-6) and unbalanced satellite (Figs. 7, 8). According to Figs. (4-6), when the satellite is principally spinning around its long axis or $\mathrm{x}$ axis, damping of un-spin angular velocity is performed exactly and the spin rate is remained constant (Fig. 5). But in unbalanced satellite not only un-spin angular velocities are damping, but also spin rate is decreased (Figs. 7, 8).

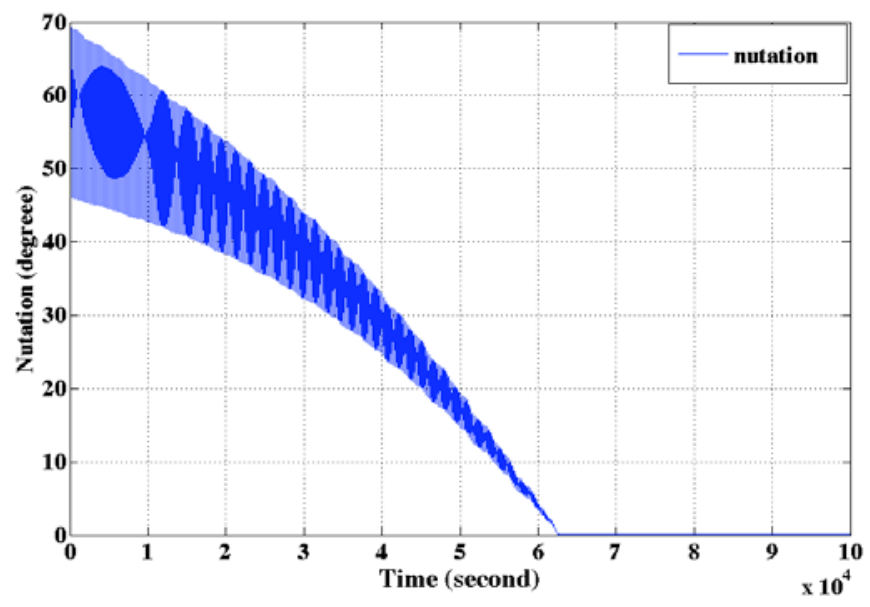

Fig. (4). Nutation damping using hysteresis damper in the dynamically balanced spin satellite.

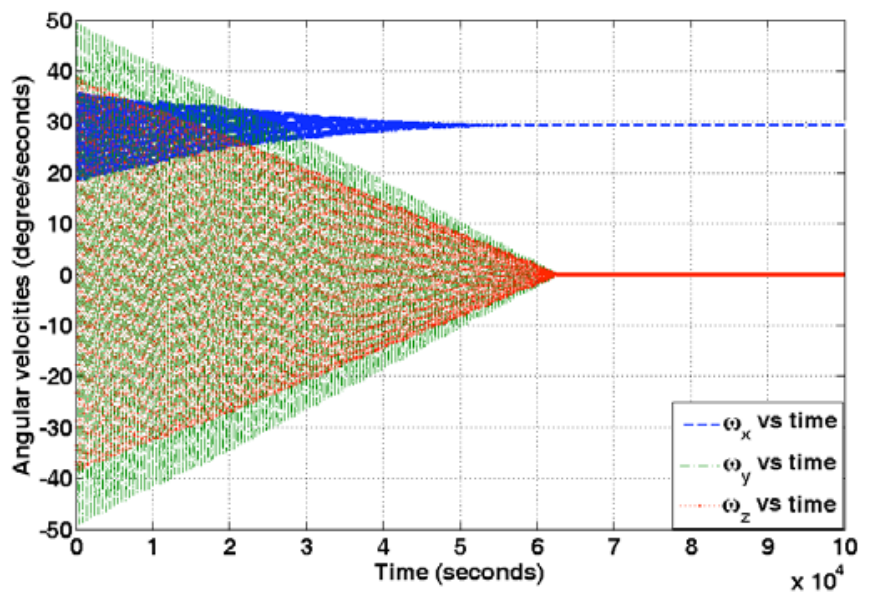

Fig. (5). Hysteresis damper effect on angular velocities in the dynamically balanced spin satellite.

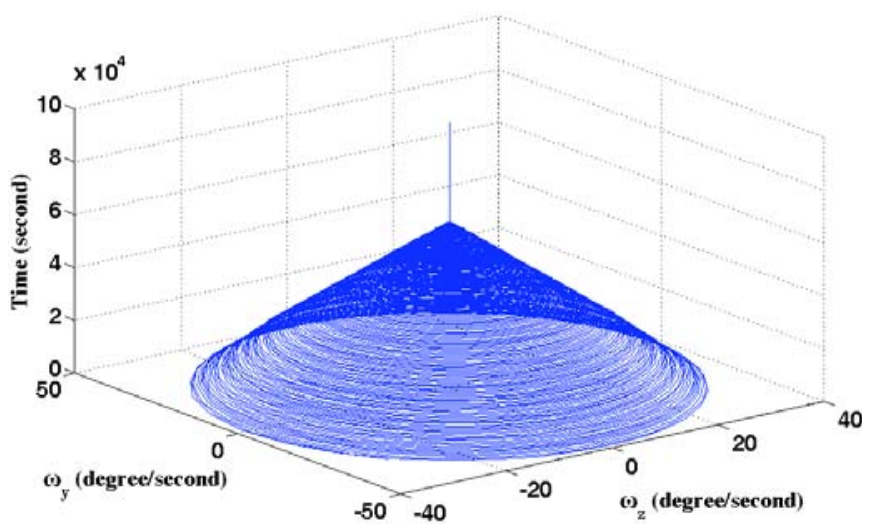

Fig. (6). Un-spin angular velocities damping using hysteresis damper in the dynamically balanced spin satellite.

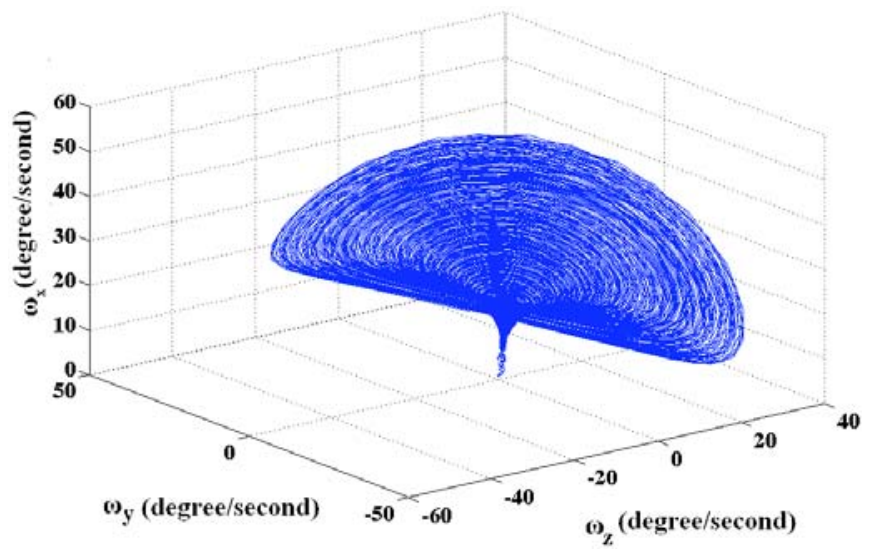

Fig. (7). Hysteresis damper behavior in the dynamically unbalanced spin satellite (Spin rate is dissipated).

\section{CONCLUSION}

This paper presented a hysteresis nutation damper for the dynamically balanced and unbalanced spinning satellites. The spin and hysteresis rods dynamic conditions were modeled. This model is a passive solution to nutation damping in spin satellites while the hysteresis rods are aligned with the satellite spin axis. Therefore, as a result of 


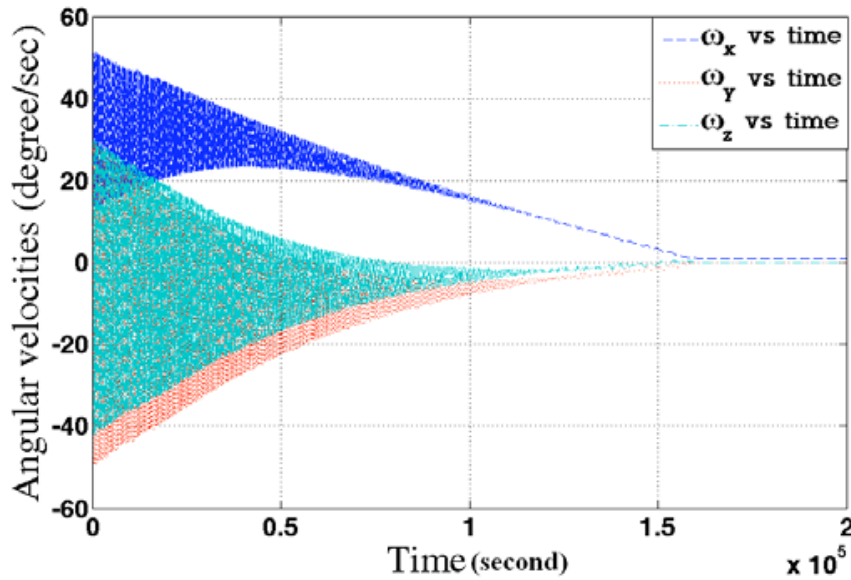

Fig. (8). All angular velocities damping in the dynamically unbalanced spin satellite (Spin rate is dissipated).

these simulations, hysteresis damper is not appropriate for dynamically unbalanced spin satellite because the spin axis is not aligned with satellite angular momentum. Consequently, in order to use hysteresis rods as nutation damper, it's necessary to dynamically balance the satellite during the ground tests.

\section{CONFLICT OF INTEREST}

The authors confirm that this article content has no conflict of interest.

\section{ACKNOWLEDGEMENTS}

Declared none.

\section{REFERENCES}

[1] J. W. Miles, "On the annular damper for a freely precessing gyroscope". J. Appl. Mech., vol. 30, pp. 189-192, 1963.

[2] K. T. Alfriend, "Partially Filled Viscous Ring Nutation Damper" J. Spacecr. Rockets, vol. 11, no. 7, pp. 456-462, July 1974.

[3] J. E. Cochran, and J. A. Thompson, "Nutation Dampers vs Precession Dampers for Asymmetric Spacecraft." $J$. Guidance,Control Dyn., vol 3, no. 1, pp. 22-28, JanuaryFebruary 1980.

[4] J. R. Alper, "Analysis of Pendulum Damper for Satellite Wobble Damping." J. Spacecr. Rockets, vol. 2, no. 1, pp.50-54, JanuaryFebruary 1965.

[5] Sidi J.M, Spacecraft Dynamics and Control. University Press, Cambridge, 1997.

[6] A.E. Chinnery, "Numerical Analysis of a Rigid Body with an Attached Spring-Mass-Damper", MS Thesis, Graduate School of Engineering, Air Force Instituteof Technology (AETC), USA, 1994.

[7] G. Park, "A Dynamic Model of a Passive Magnetic Attitude Control System for the RAX Nanosatellite" In: AIAA Guidance, Navigation, and Control Conference, 2 - 5 August 2010, Toronto, Ontario Canada, AIAA, 2010.

[8] F. M. Poppenk and R. Amini, "Delfi-C3 Control System Development and verification", In: IAC-06-C1.2.02.

[9] V. F.-Lavet, "Study of passive and active attitude controlsystems for the OUFTI nanosatellites", Master thesis. Engineering Physics, University of Liège, Belgium, 2009-2010

[10] T.W. Flatley, and D. A. Henretty, "A Magnetic Hysteresis Model," Tech. Rep. N95-27801, NASA GSFC, USA, 1995.

[11] R.R. Kumar, D.D. Mazanek, and M.L. Heck, "Simulation and Shuttle Hitchhiker Validation of Passive Satellite Aero stabilization", J. Spacecr. Rockets, vol. 32, no. 5, 1995.

[12] H. S. Oosaloo, "Magnetic Attitude Control of Dynamically Unbalanced Spinning Spacecraft during Orbit Raising" J. Aerospace engin. ASCE, 2012.

[13] D. Pais, and S. Jayaram, "Satellite Passive Attitude Stabilization using Permanent Magnets" Dynamic Model and Simulation, Saint Louis University, USA, 2006 